\title{
XMM-Newton survey of the Local Group galaxy M 33 - bright individual sources
}

\author{
Z. Misanovic ${ }^{1}$, W. Pietsch ${ }^{1}$, F. Haberl ${ }^{1}$, G. Trinchieri ${ }^{2}$, M. Ehle ${ }^{3}$, \\ and D. Hatzidimitriou ${ }^{4}$ \\ ${ }^{1}$ Max-Planck-Institut für extraterrestrische Physik, Giessenbachstraße, 85741 Garching, \\ Germany \\ email: zdenka@mpe.mpg.de, wnp@mpe.mpg.de, fwh@mpe.mpg.de \\ ${ }^{2}$ Osservatorio Astronomico di Brera, via Brera 28, 20121 Milano, Italy \\ email: ginevra@brera.mi.astro.it \\ ${ }^{3}$ XMM-Newton Science Operations Centre, ESAC, ESA, P.O. Box 50727, 28080 Madrid, Spain \\ email: Matthias.Ehle@sciops.esa.int \\ ${ }^{4}$ Department of Physics, University of Crete, P/O. Box 2208, 71003 Heraklion, Crete, Greece \\ email: dh@physics.uoc.gr
}

\begin{abstract}
As shown in our first poster, in a recent survey of M 33 with XMM-Newton we detected the X-ray source population of this nearby spiral galaxy down to the $(0.2-4.5) \mathrm{keV}$ luminosity of $10^{35} \mathrm{erg} \mathrm{s}^{-1}$, a factor of 10 deeper than in previous observations. The majority of the detected sources was classified using, in many cases, only their X-ray properties. In particular, 8 new X-ray binary (XRB) candidates were selected, based on their long-term X-ray light curves. We also classified supernova remnants (SNRs), super-soft sources (SSS), AGN, foreground stars and a population of 'hard' sources using the hardness ratio (HR) method. A detailed spectral and timing analysis of the brightest sources is in progress. We present a few examples of spectra for particular source classes. We find that bright 'hard' sources can be divided into two broad families: one best modelled by a powerlaw with photon index in the range of $1.0-2.0$, and the other displaying disk blackbody spectra with $\mathrm{kT}$ of 0.8 to $1.5 \mathrm{keV}$.
\end{abstract}

Keywords. X-rays: galaxies, X-rays: binaries.

\section{Introduction}

As demonstrated by Pietsch et al. (2004a), we can use HRs to distinguish very soft, soft and hard sources. SNRs and foreground stars exhibit soft spectra with the emission mainly below $1.0 \mathrm{keV}$, while SSS have extremely soft spectra (below $0.5 \mathrm{keV}$ ). XRBs, Crab-like SNRs and background AGN have significantly harder spectra. We perform detailed spectral and timing analysis of the brightest sources in all individual XMMNewton observations of M33. Below we show several examples of typical source spectra.

\section{Source spectra}

The first source in Fig. 1 is a SNR, which we fitted by a two-temperature plasma (MEKAL) model and an absorption column of $6 \times 10^{20} \mathrm{~cm}^{-2}$. The variable source 253 also requires a two component model (MEKAL+POWERLAW). This source is identified as a late G-type star in optical follow up observations (Hatzidimitriou et al. 2005), and its relatively hard spectrum can, most probably, be attributed to flaring, although we do not have enough counts in any of the individual observations to confirm this. We fit an extremely variable SSS by an absorbed black body model with a kT of $62 \mathrm{eV}$. 

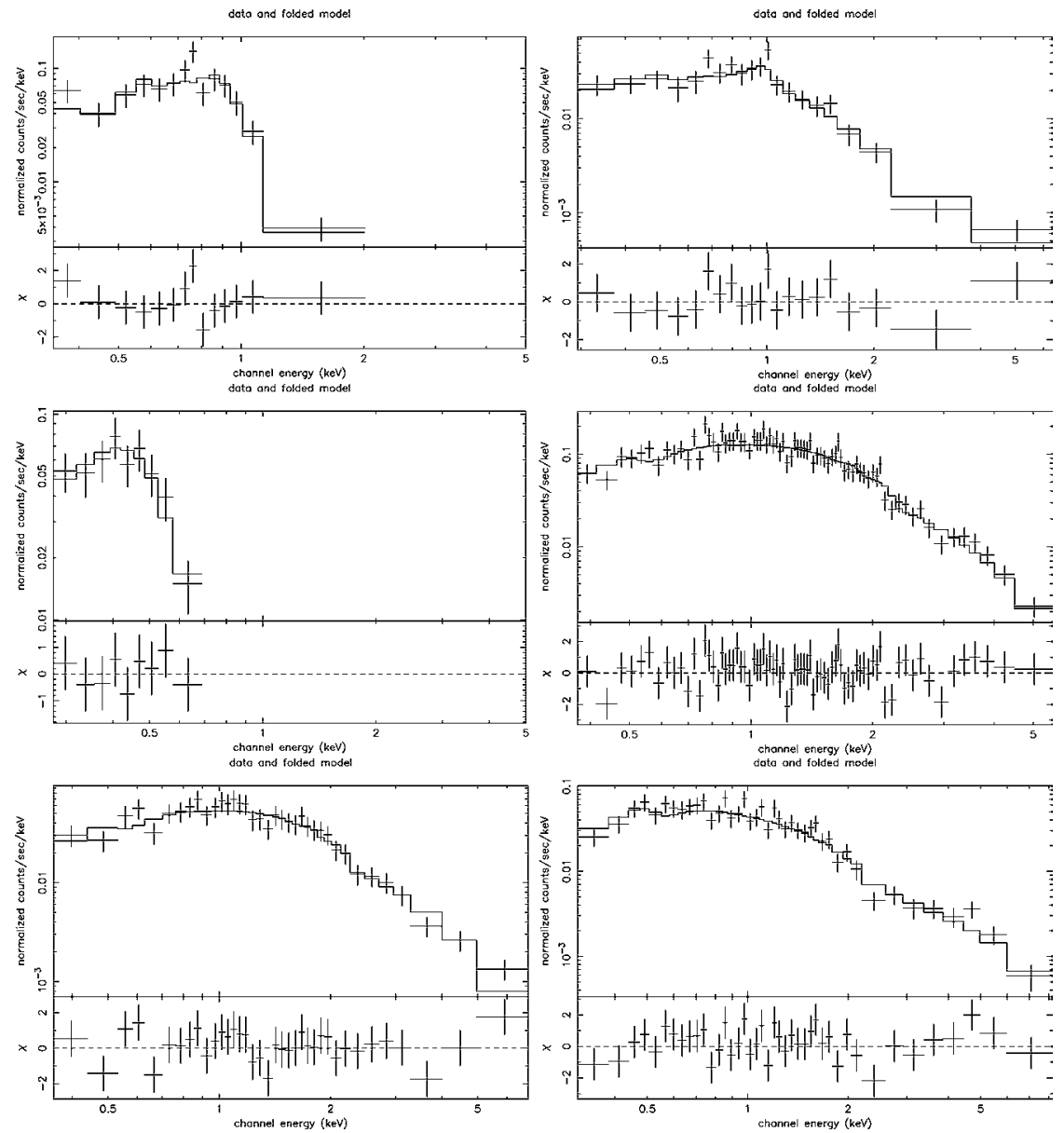

Figure 1. Spectra of bright X-ray sources detected in XMM-Newton survey of M 33. From upper left to lower right: Src 108 (SNR), 253 (fgStar), 207 (SSS), 150 (XRB M 33 X-7), 124 ('hard') and 248 (AGN). The source number and classification are from Misanovic et al. (2005).

The remaining sources from our selection display hard spectra. For M 33 X-7, a known eclipsing high mass XRB (see e.g. Pietsch et al. 2004b), we extract a spectrum in the high state and fit it by an absorbed disk blackbody with an intrinsic absorption column of about $3 \times 10^{20} \mathrm{~cm}^{-2}$ and a kT of $0.89 \mathrm{keV}$. A 'hard' source (Src 124) has a similar spectral shape - almost the same absorption and a temperature of $1.03 \mathrm{keV}$. The powerlaw model also gave acceptable fits for these two sources, however, requiring significantly higher absorption columns and spectral indices in the range 2.6-3.0.

We fit a source classified as an AGN and a 'hard' source (SRC 131) with the powerlaw models with spectral indices of 2.0 and 1.6 respectively.

\section{References}

Hatzidimitriou, D., Pietsch, W., Misanovic, Z., Rieg, P. \& Haberl, F. 2005, A\&A, in preparation Misanovic, Z., Pietsch, W., Haberl, F., et al. 2005, A\&A, in press

Pietsch, W., Misanovic, Z., Haberl, F., et al., 2004a, A\&A, 426, 11

Pietsch, W., Mochejska, B.J., Misanovic, Z., et al. 2004b, A\&A, 413, 871 